\title{
Assessment of consciousness with electrophysiological and neurological imaging techniques
}

\author{
Marie-Aurélie Bruno ${ }^{\mathrm{a}, *}$, Olivia Gosseries ${ }^{\mathrm{a}, *}$, Didier Ledoux ${ }^{\mathrm{a}}$, Roland Hustinx ${ }^{\mathrm{b}}$ and \\ Steven Laureys ${ }^{\mathrm{a}}$
}

\begin{abstract}
${ }^{a}$ Coma Science Group, Cyclotron Research Centre, University of Liège and ${ }^{\mathrm{b}}$ Department of Nuclear

Medicine, University Hospital of Liège, Liège, Belgium

Correspondence to Dr Steven Laureys, Coma Science Group, Cyclotron Research Center, University of Liège, Sart-Tilman B30, 4000 Liège, Belgium

Tel: +32 436623 16; fax: +32 436629 46;

e-mail: steven.laureys@ulg.ac.be

* Marie-Aurélie. Bruno and Olivia Gosseries contributed equally to the writing of this article.
\end{abstract}

Current Opinion in Critical Care 2011, 17:000-000

\author{
Purpose of review \\ Brain MRI (diffusion tensor imaging and spectroscopy) and functional neuroimaging \\ (PET, functional MRI, EEG and evoked potential studies) are changing our \\ understanding of patients with disorders of consciousness encountered after coma \\ such as the 'vegetative' or minimally conscious states. \\ Recent findings \\ Increasing evidence from functional neuroimaging and electrophysiology demonstrates \\ some residual cognitive processing in a subgroup of patients who clinically fail to show \\ any response to commands, leading to the recent proposal of 'unresponsive \\ wakefulness syndrome' as an alternative name for patients previously coined \\ 'vegetative' or 'apallic'.
}

Summary

Consciousness can be viewed as the emergent property of the collective behavior of widespread thalamocortical frontoparietal network connectivity. Data from physiological, pharmacological and pathological alterations of consciousness provide evidence in favor of this hypothesis. Increasing our understanding of the neural correlates of consciousness is helping clinicians to do a better job in terms of diagnosis, prognosis and finally treatment and drug development for these severely brain-damaged patients. The current challenge remains to continue translating this research from the bench to the bedside. Only well controlled large multicentric neuroimaging and electrophysiology studies will enable to identify which paraclinical diagnostic or prognostic test is necessary for our routine evidence-based assessment of individuals with disorders of consciousness.

\author{
Keywords \\ $\mathrm{PET}$, vegetative state/unresponsive wakefulness syndrome \\ Curr Opin Crit Care 17:000-000 \\ (C) 2011 Wolters Kluwer Health | Lippincott Williams \& Wilkins \\ 1070-5295
}

consciousness, electroencephalography, functional MRI, minimally conscious state,

\section{Introduction}

We will here review the medical (i.e. diagnostic, prognostic, and therapeutic) and scientific interest of neuroimaging and electrophysiological studies in disorders of consciousness (DOC) $\left[1^{\bullet}\right]$. Following severe brain damage and coma, some patients may awaken (i.e. open the eyes) but remain unresponsive (i.e. only showing reflex movements). In Europe, this clinical syndrome was initially termed apallic syndrome or coma vigil, but it was later redefined as vegetative state [2]. Since its description more than 35 years ago, an increasing number of functional neuroimaging and event related potential studies (e.g. for recent studies see $[3,4]$ ) have shown that it sometimes may be difficult to make strong claims about 'vegetative' patients' awareness [5]. This situation is further complicated when patients have underlying deficits in verbal or nonverbal communication functions, such as aphasia, agnosia or apraxia $\left[6^{\bullet}, 7^{\bullet}\right]$. It appears that part of the healthcare, media and lay public continues to feel some unease regarding the unintended denigrating 'vegetable-like' connotation seemingly intrinsic to the term vegetative state. The European Task Force on Disorders of Consciousness therefore recently proposed an alternative name: 'unresponsive wakefulness syndrome' (UWS) [ $\left.8^{\bullet}\right]$. Hence, physicians are recently offered to refer to these patients as UWS, a more neutral and descriptive term, pertaining to patients showing a number of clinical signs (hence syndrome) of unresponsiveness (i.e. without response to commands or oriented voluntary movements) in the presence of wakefulness. In contrast to coma, which is an acute condition lasting no more than some days or weeks, vegetative state/UWS can be a chronic condition lasting years or 
Figure 1 Clinical criteria of disorders of consciousness

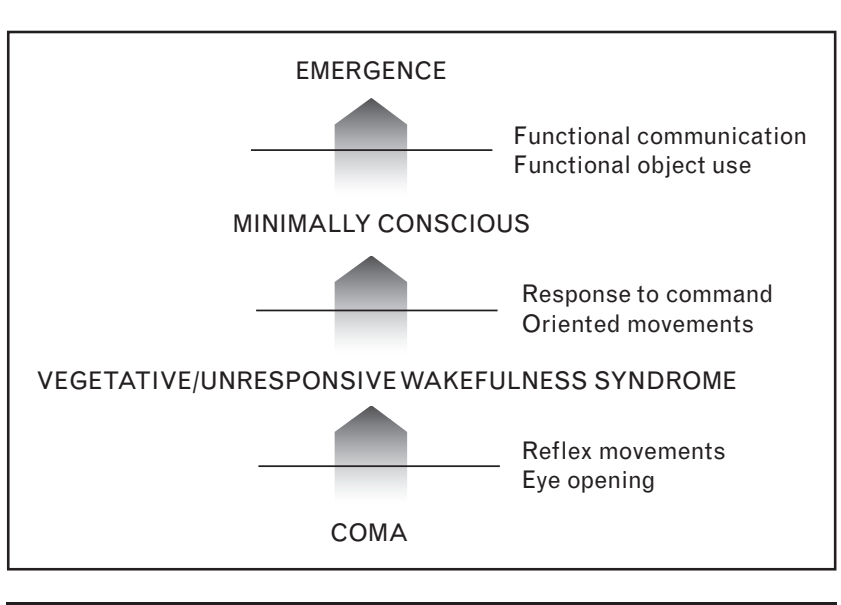

remain permanent. Patients who do recover, classically evolve to a minimally conscious state (MCS), defined by the presence of nonreflex voluntary movements such as orientation to pain, eye tracking or reproducible albeit inconsistent command following. By definition, MCS patients cannot communicate their thoughts or wishes [9]. Finally, in pseudocoma or locked-in syndrome, patients may awaken from coma fully conscious but paralyzed, only able to communicate by eye movements. The medical and ethical management of these vulnerable patients is very complex and a recent article reviewed the even more challenging (but fortunately very rare) situation of locked-in syndrome in children $\left[10^{\circ}\right]$. Locked-in patients illustrate how difficult it can be to make inferences about subjective conscious awareness in severely motor impaired brain lesioned patients. How can we measure consciousness at the bedside (Fig. 1)?

\section{Quantifying consciousness}

The clinical assessment of consciousness relies on disentangling automatic responses from nonreflex-oriented movements or command following. This can be very challenging in coma and related disorders. Motor responses may be very small, inconsistent and easily exhausted, potentially leading to diagnostic errors [11]. A recent prospective study on 103 coma survivors showed that the clinical consensus diagnosis of 'vegetative state/ UWS', attributed to 44 patients, was incorrect in 18 cases when properly assessed using a standardized consciousness scale, the Coma Recovery Scale-Revised [12 $\bullet$ ]. Such a high rate of diagnostic error (i.e. 41\%) should prompt clinicians to use validated behavioral scales of consciousness before making the diagnosis of vegetative state/UWS.

Similar to the problem of quantifying consciousness, it remains a clinical and ethical challenge to measure con-

\section{Key points}

- Consciousness can be viewed as the emergent property of the collective behavior of widespread thalamocortical frontoparietal network connectivity.

- Electrophysiological and neuroimaging assessments are increasing our understanding of the neural correlates of consciousness and hence permitting to improve diagnosis, prognosis and management of coma and related disorders.

- Recent studies have demonstrated some residual cognitive processing in a subgroup of patients who clinically fail to show any response to commands, leading to the recent proposal of 'unresponsive wakefulness syndrome' as an alternative name for patients previously coined 'vegetative' or 'apallic'.

- 'The current challenge remains to continue translating this research from the bench to the bedside offering large controlled multicentric studies to enable identification of evidence-based paraclinical diagnostic and prognostic tests in patients with disorders of consciousness.

scious perception of pain in these patients with DOC. A European survey showed that $56 \%$ of 1166 medical and $68 \%$ of 538 paramedical professionals thought that 'vegetative' patients could feel pain $\left[13^{\circ}\right]$. Healthcare givers with religious affiliation reported more often that vegetative state/UWS patients might experience pain. Different beliefs on possible pain perception may have consequences in terms of analgesic treatment. When the decision to treat for pain is taken, it remains difficult to monitor the therapeutic effect in these noncommunicative patients. Schnakers et al. $\left[14^{\bullet}\right]$ therefore proposed a new scale to standardize and evaluate nociception in DOC, the Nociception Coma Scale. When patients score more than 7 on a total score of 12 , the authors propose to start analgesia. Objective paraclinical neuroimaging studies are needed to increase our understanding of residual sensation in DOC patients and to propose evidencebased medical guidelines for their care $\left[15^{\bullet}\right]$.

\section{Paraclinical diagnostic markers}

Structural MRI studies such as diffusion tensor imaging permit to quantify lesions to the brain's white matter tracts, often invisible to conventional radiological approaches [16]. Fernandez-Espejo et al. $\left[17^{\bullet}\right]$ used this technique in 25 DOC patients of traumatic and nontraumatic cause and showed that vegetative state/UWS could be differentiated from MCS with a $95 \%$ accuracy.

Functional imaging such as PET permits to quantify the brain's glucose metabolism in 'resting' (i.e. unstimulated) conditions. Phillips et al. [18 $\left.{ }^{\bullet \bullet}\right]$ recently developed a 
'relevance vector machine' automated consciousness classifier using metabolic PET scans to categorize, with a $100 \%$ accuracy, eight conscious locked-in from 13 unconscious vegetative state/UWS patients. Cerebral PET imaging can also be employed to disentangle reflex from nonreflex movements. Bruno et al. $\left[19^{\circ}\right]$ showed that anoxic vegetative state/UWS patients with $(n=5)$ or without $(n=5)$ visual fixation presented an identical brain metabolism and corticocortical functional connectivity, concluding that visual fixation does not necessarily reflect consciousness.

Resting state functional MRI similarly can measure the brain's 'default' activity looking at spontaneous hemodynamic fluctuations in blood-oxygen-level-dependent (BOLD) signal. It has long remained controversial whether these spontaneous fluctuations reflect consciously directed mental activity. However, recent evidence shows that this technique can be used to correlate subjective 'internal' self-related thoughts with activity in midline cortical structures and 'external' sensory perceptions with lateral frontoparietal activity [20]. If this is true, it would be useful to assess fMRI 'resting state' brain fluctuations, considered to reflect spontaneous thoughts, in coma and 'vegetative' patients - who are not supposed to have any thoughts or feelings. Boly et al. [21] were the first to use this approach in brain death and vegetative state/UWS, showing a complete absence of 'default mode network' connectivity in the former and a partially preserved activity in the latter condition. Building on these results, Vanhaudenhuyse et al. [22*•] studied resting-state fMRI connectivity in 14 severely brain-damaged patients of mixed cause, reporting a nonlinear disintegration when conscious locked-in syndrome patients were compared to MCS, vegetative state/UWS and comatose states. Similarly, dysfunctional 'default mode network' connectivity was reported by others in small case series of vegetative state/UWS $[23,24]$, but its diagnostic value at the individual patient level remains to be shown.

fMRI activation studies classically measure the brain's response to external sensory stimuli. Qin et al. [25] has studied auditory processing in 11 DOC patients using an autoreferential attention-grabbing stimulus, that is the patient's own name. They reported a linear correlation between activation of the limbic system (anterior cingulate cortex) and the level of consciousness as quantified by the Coma Recovery Scale-Revised. However, such 'passive' stimulation-induced brain activation does permit to make strong claims about the patient's consciousness.

Monti et al. $\left[26^{\bullet \bullet}\right]$ used a so called 'active' paradigm in which patients were instructed to follow simple commands. Their study enrolled 54 DOC cases of different cause in imaging centers in Cambridge and Liège, asking patients to perform two mental imagery tasks: 'imagine playing tennis' (activating motor areas) and 'imagine to walk around in your house' (activating parahippocampal brain regions). Of the 31 patients previously diagnosed as MCS based on standardized behavioral assessments, only one was able to show reliable fMRI activation in the expected brain areas, illustrating that the employed technique only has a sensitivity of $3 \%$ in minimally conscious DOC patients (i.e. has a $97 \%$ false negative rate). It is more difficult to calculate the technique sensitivity and specificity in vegetative state/UWS patients, as there is no gold standard for assessing consciousness. Indeed, in the published case series, four out of 23 clinically 'unconscious' patients showed fMRI signs of command following and hence of consciousness (i.e. 17\%). Nevertheless, given the known low sensitivity, it could be that other patients might have been (minimally) conscious, yet were unable to do the task or show statistically significant brain activation. However, in those individual patients in whom reliable and long-lasting (>30 s) command-related fMRI activation was observed, there seems no other logical alternative explanation other than that they repeatedly understood and replied to the commands and hence truly showed conscious awareness [27,28]. Similarly, a recent study by Rodrigez-Moreno et al. [29] used a silent picture-naming task as an 'active' fMRI paradigm searching for proof of consciousness in 10 DOC patients of mixed cause.

In addition to demonstrate proof of consciousness, fMRI can now be used to communicate with some (very exceptional) DOC patients. Indeed, one clinically noncommunicative patient studied in the Liège University Hospital was shown to correctly answer five out of six simple questions regarding his family members' names [26 $\left.{ }^{\bullet \bullet}\right]$. Here, it was set that to communicate 'yes' one should imagine playing tennis (and hence motor area activation was identified using an automated user-independent relative-similarity classifier) and to communicate 'no' the spatial navigation task should be performed (activating parahippocampal areas). fMRI muscle-independent communication currently even goes beyond such yes-no answers to opened questions and is offering the possibility to answer multiple choice questions [ $\left[30^{\circ}\right]$. Evidently, these data should be seen as proofs of concept rather than as a practical means to truly assure long-term communication. EEG-based communication devices, called brain-computer interfaces, are therefore being developed as a more practical, transportable and cheaper alternative.

Similarly to the described fMRI 'active' paradigms, EEG-based technology will aim to answer two questions: 'Is the patient conscious?' and, if the answer is affirmative; 'What is that consciousness like?', needing functional communication [31]. The practical utility of demonstrating voluntary brain activity using nonclinical 
means was recently illustrated in the case of a 21-year-old comatose woman who failed to show any motor sign of conscious awareness up to 49 days after an extensive brainstem and thalamic stroke [32]. Only EEG evoked potential based on command following (i.e. counting a target name in a list of names) permitted to make the diagnosis of complete locked-in syndrome shown at the ICU. Similarly, EEG-based evidence of conscious processing (i.e. count a deviant sound sequence in a series of beeps) was demonstrated in three out of four MCS patients while no 'willful' modulation of evoked potentials was recorded in four vegetative state/UWS patients [33]. Ongoing multicentric trials are validating the possible prognostic value of these tests.

\section{Paraclinical tests predicting outcome}

Structural MRI diffusion tensor imaging combined with MRI spectroscopy holds promising potential as an objective quantitative outcome-prediction tool $\left[34,35^{\circ}\right]$. This method provides useful metabolic information on brain damage that may not be visible on classical morphologic imaging. In a cohort of 43 DOC patients studied 2-5 weeks after a traumatic brain injury, the combination of both MRI measurements permitted to predict 1-year unfavorable outcome with up to $86 \%$ sensitivity and $97 \%$ specificity $\left[36^{\circ}\right]$. This approach is also being employed in vegetative state/UWS [37] and MCS patients [38]. The prognostic value of MRI spectroscopy was also shown in 90 children with nonaccidental trauma $\left[39^{\circ}\right]$.

The prognostic value of functional MRI 'passive' activation studies was proposed by Di et al. [40] concluding that vegetative state/UWS patients with absent or 'lowlevel' sensory cortex activation showed 100 and $84 \%$ of bad outcome, respectively, whereas those showing 'higher-level' associative cortex activation showed a $82 \%$ recovery rate (i.e. resulting in a $93 \%$ specificity and $69 \%$ sensitivity of the included 14 studies encompassing 48 patients). A prospective study using auditory stimulation in 22 vegetative state/UWS and 19 MCS patients recently confirmed the predictive value of fMRI activation studies by demonstrating that the level of cerebral processing correlated with patients' recovery at 6 months follow-up $\left[41^{\circ}\right]$.

Similarly, electrophysiological studies have aimed to predict outcome from DOC. A standardized classification method of routine 'resting-state' EEG showed a correlation at the group level with 3-month outcome measures in 46 DOC patients of varying cause [42]. In a retrospective study of 'resting-state' routine EEG from 50 vegetative state/UWS patients, Babiloni et al. [43] showed that increased alpha wave power correlated with 3 -month recovery. Cologan et al. [44$\left.{ }^{\bullet}\right]$ recently reviewed the literature on sleep-wake EEG recordings in DOC, discussing the positive predictive value of sleep 'spindle' waves for recovery of consciousness. Using evoked potentials and 'passive' auditory oddball stimuli, the presence of a 'P300' wave to rare stimuli correlated with recovery of consciousness in 34 patients with posttraumatic vegetative state/UWS [45]. Finally, the presence of Pavlovian eye-puff trace conditioning was proposed as a marker of learning in 20 DOC patients, correlating with recovery $\left[46^{\bullet \bullet}\right]$. In addition to their clinical diagnostic and prognostic usefulness, these studies have evident neuroscientific importance, helping in our understanding of the neural correlates of human consciousness.

\section{Conclusion}

Consciousness can be viewed as the emergent property of the collective behavior of widespread thalamocortical frontoparietal network connectivity [47]. Many of the above-presented studies, and increasing evidence from neuroscientific studies not discussed here on physiological (e.g. sleep $\left[48^{\bullet}, 49\right]$ and hypnosis $[50,51]$ ), pharmacological (e.g. in general anesthesia $\left.\left[52^{\circ}\right]\right)$ and pathological alterations of consciousness (e.g. $\left[53,54,55^{\bullet}, 56^{\bullet}\right]$ ) provide evidence in favor of this hypothesis. For most neuroscientists, you are your brain. However, such a scientific perspective of human consciousness, awareness or mind is not universally accepted. A recent survey of 782 medical and 290 paramedical professionals showed that more than one-third regarded mind and brain as separate entities. Such dualistic perceptions were predicted most strongly by personal religious and philosophical convictions [57]. The study of consciousness has indeed been the subject of philosophy for centuries. Functional neuroimaging and electrophysiology has now changed this, permitting to make measurements of the brain's activity and correlate this with conscious perception in health and disease. This short review on last year's articles in the field illustrates the increase in our understanding of the neural correlates of consciousness. This knowledge helps clinicians to do a better job in terms of diagnosis [58], prognosis [59] and finally, we hope, treatment [60] and may improve industry drug development (e.g. the use of functional imaging in demonstrating the effect of amantadine [61] and methylphenidate [62] on 'consciousness' networks in DOG patients). Increasing evidence from functional neuroimaging and electrophysiology demonstrates some residual cognitive processing in a subgroup of patients who clinically fail to show any response to commands. This led to the recent proposal of 'unresponsive wakefulness syndrome' as an alternative name for patients previously coined 'vegetative' or 'apallic'. The current challenge remains to continue translating this research from the bench to the bedside - within a well defined ethical framework (e.g. see [63-65]). Only well controlled large multicentric neuroimaging and 
electrophysiology studies will enable to identify which paraclinical diagnostic or prognostic test is necessary, at any given time, for our routine evidence-based assessment of individuals with DOG.

\section{Acknowledgements}

This study was supported by the Fonds de la Recherche Scientifique (FNRS), James S. McDonnell Foundation, Mind Science Foundation, European Commission (Mindbridge, DISCOS, DECODER and COST) and Concerted Research Action (ARC 06/11-340). M.A.B. and O.G. are Research Fellow at FNRS. D.L. is Postdoctoral Fellow at FNRS. S.L. is Senior Research Associate at FNRS.

\section{References and recommended reading}

Papers of particular interest, published within the annual period of review, have been highlighted as:

- of special interest

-• of outstanding interest

Additional references related to this topic can also be found in the Current

World Literature section in this issue (pp. 000-000).

1 Bernat JL. Chronic consciousness disorders. Annu Rev Med 2009; 60:381- 392.

Outstanding authoritative review of the 'current state of the art' in clinical and paraclinical assessment of consciousness in coma and related conditions.

2 Jennett B. Thirty years of the vegetative state: clinical, ethical and legal problems. Prog Brain Res 2005; 150:537-543.

3 Fischer C, Luaute J, Morlet D. Event-related potentials (MMN and novelty P3) in permanent vegetative or minimally conscious states. Clin Neurophysiol 2010; 121:1032-1042.

4 Kotchoubey B, Kaiser J, Bostanov V, et al. Recognition of affective prosody in brain-damaged patients and healthy controls: a neurophysiological study using EEG and whole-head MEG. Cogn Affect Behav Neurosci 2009; 9:153-167.

5 Monti MM, Laureys S, Owen AM. The vegetative state. Br Med J 2010; 341:292-296.

6 Majerus S, Bruno MA, Schnakers C, et al. The problem of aphasia in the

- assessment of consciousness in brain-damaged patients. Prog Brain Res $2009 ; 177: 49-61$.

This article addresses the difficulties of making inferences about consciousness when looking for command following (at the bedside or using fMRI) in braindamaged patients who may have speech comprehension problems.

7 Bruno MA, Fernández-Espejo D, Lehembre R, et al. Multimodal neuroimaging

- in patients with disorders of consciousness showing 'functional hemispherectomy'. Prog Brain Res (in press).

Illustration of the complementarity of PET, fMRI resting state, high-density EEG and diffusion tensor imaging MRI in the assessment of patients with DOC.

8 Laureys S, Celesia G, Cohadon F, et al. Unresponsive wakefulness syndrome:

- a new name for the vegetative state or apallic syndrome. BMC Med 2010; 8:68.

'Unresponsive wakefulness syndrome' is here proposed by the European Task Force on Disorders of Consciousness as an alternative name for the 'vegetative state' an over 35-year-old syndrome with an unintended albeit persistently negative connotation.

9 Giacino JT, Schnakers C, Rodriguez-Moreno D, et al. Behavioral assessment in patients with disorders of consciousness: gold standard or fool's gold? Prog Brain Res 2009; 177:33-48.

10 Bruno MA, Schnakers C, Damas F, et al. Locked-in syndrome in children:

- report of five cases and review of the literature. Pediatr Neurol 2009; 41:237246.

Discussion of the medical and ethical challenges encountered in five cases of locked-in syndrome in children.

11 Monti MM, Coleman MR, Owen AM. Neuroimaging and the vegetative state: resolving the behavioral assessment dilemma? Ann N Y Acad Sci 2009; 1157:81-89.

12 Schnakers C, Vanhaudenhuyse A, Giacino J, et al. Diagnostic accuracy of the

- vegetative and minimally conscious state: clinical consensus versus standardized neurobehavioral assessment. BMC Neurol 2009; 9:35.

A milestone study confirming the high misdiagnosis rate in vegetative state/UWS, apparently unchanged over the past 15 years and demonstrating the usefulness of standardized validated behavioral assessment tools as compared to unstructured routine neurological assessment in DOC.
13 Demertzi A, Schnakers C, Ledoux D, et al. Different beliefs about pain - perception in the vegetative and minimally conscious states: a European survey of medical and paramedical professionals. Prog Brain Res 2009; 177:329-338.

Important survey assessing healthcare givers' beliefs on possible pain perception in DOC and discussing its impact on treatment options.

14 Schnakers C, Chatelle C, Vanhaudenhuyse A, et al. The Nociception Coma

- Scale: a new tool to assess nociception in disorders of consciousness. Pain 2010; 148:215-219.

Presentation of a new bedside scale permitting to standardize the evaluation and monitoring of pain in noncommunicative brain-damaged patients - its real clinical usefulness awaits to be demonstrated.

15 Owen AM, Schiff ND, Laureys S. A new era of coma and consciousness - science. Prog Brain Res 2009; 177:399-411.

Authoritative review of the field's pioneers on the rapid technological developments in the field of neuroimaging producing a cornucopia of new techniques for examining both the structure and function of the human brain in vivo in DOC.

16 Newcombe VF, Williams GB, Scoffings D, et al. Aetiological differences in neuroanatomy of the vegetative state: insights from diffusion tensor imaging and functional implications. J Neurol Neurosurg Psychiatry 2010; 81:552-561.

17 Fernandez-Espejo D, Bekinschtein T, Monti MM, et al. Diffusion weighted - imaging distinguishes the vegetative state from the minimally conscious state. Neuroimage 2010; 1:103-112.

An original study on the diagnostic role of MRI white matter tract integrity assessment in DOC.

18 Phillips $C L$, Bruno MA, Maquet $P$, et al. 'Relevance vector machine' con-. sciousness classifier applied to cerebral metabolism of vegetative and lockedin patients. Neuroimage 2010.

Illustration of the usefulness of an automated user-independent classifier disentangling unconscious from 'locked-in' brain damaged patients using cerebral PET metabolic imaging, outperforming the human eye.

19 Bruno MA, Vanhaudenhuyse A, Schnakers C, et al. Visual fixation in the - vegetative state: an observational case series PET study. BMC Neurol 2010; 10:35.

A interesting novel approach of correlating specific behavioral signs in DOC with functional neuroimaging results - hence helping to identify their underlying functional neuroanatomy and possible reflection of conscious awareness.

20 Vanhaudenhuyse A, Demertzi A, Schabus M, et al. Two distinct neuronal networks mediate the awareness of environment and of self. J Cogn Neurosci 2011; 23:570-578.

21 Boly M, Tshibanda L, Vanhaudenhuyse A, et al. Functional connectivity in the default network during resting state is preserved in a vegetative but not in a brain dead patient. Hum Brain Mapping 2009; 30:2393-2400.

22 Vanhaudenhuyse A, Noirhomme Q, Tshibanda LJ, et al. Default network -- connectivity reflects the level of consciousness in noncommunicative braindamaged patients. Brain 2010; 133:161-171.

'The brain never rests' as illustrated by this 'resting state' fMRI study in locked-in, minimally conscious, unresponsive and comatose patients showing the potential diagnostic value of recording the brain's activity without any stimulation or task currently awaiting assessment of its possible prognostic value.

23 Cauda F, Micon BM, Sacco K, et al. Disrupted intrinsic functional connectivity in the vegetative state. J Neurol Neurosurg Psychiatry 2009; 80:429-431.

24 Bruno MA, Soddu A, Demertzi A, et al. Disorders of consciousness: moving from passive to resting state and active paradigms. Cogn Neurosci 2010; $1: 193-203$

25 Qin $\mathrm{P}, \mathrm{Di} \mathrm{H}$, Liu Y, et al. Anterior cingulate activity and the self in disorders of consciousness. Hum Brain Mapping 2010; 31:1993-2002.

26 Monti MM, Vanhaudenhuyse A, Coleman MR, et al. Willful modulation of brain - activity in disorders of consciousness. N Engl J Med 2010; 362:579-589. Milestone article on the potential of functional MRI to bridge the possible - albeit infrequent - dissociation between behavior that is observable during clinical assessment and the actual level of residual cognitive function and communication as offered by fMRI.

27 Soddu A, Boly M, Nir Y, et al. Reaching across the abyss: recent advances in functional magnetic resonance imaging and their potential relevance to disorders of consciousness. Prog Brain Res 2009; 177:261-274.

28 Stins JF, Laureys S. Thought translation, tennis and Turing tests in the vegetative state. Phenomenol Cogn Sci 2009; 8:361-370.

29 Rodriguez Moreno D, Schiff ND, Giacino J, et al. A network approach to assessing cognition in disorders of consciousness. Neurology 2010; 75:1871-1878

30 Sorger B, Dahmen B, Reithler J, et al. Another kind of 'BOLD Response':

- answering multiple-choice questions via online decoded single-trial brain signals. Prog Brain Res 2009; 177:275-292.

A study showing fMRI brain computer interface 'multiple-choice' communication in healthy volunteers - awaiting validation in DOC. 
31 Millan JD, Rupp R, Muller-Putz GR, et al. Combining brain-computer interfaces and assistive technologies: state-of-the-art and challenges. Front Neurosci 2010; 4:1-15.

32 Schnakers C, Majerus S, Goldman S, et al. Cognitive function in the locked-in syndrome. J Neurol 2008; 255:323-330.

33 Bekinschtein TA, Dehaene S, Rohaut B, et al. Neural signature of the conscious processing of auditory regularities. Proc Natl Acad Sci U S A 2009; 106:1672-1677.

34 Tshibanda L, Vanhaudenhuyse A, Galanaud D, et al. Magnetic resonance spectroscopy and diffusion tensor imaging in coma survivors: promises and pitfalls. Prog Brain Res 2009; 177:215-229.

35 Tshibanda L, Vanhaudenhuyse A, Boly M, et al. Neuroimaging after coma. - Neuroradiology 2010; 52:15-24.

Timely review on advanced MRI techniques such as spectroscopy, diffusion tensor imaging and 'activation' and 'resting-state' functional MRI that were recently introduced in the assessment of patients with disorders of consciousness.

36 Tollard E, Galanaud D, Perlbarg V, et al. Experience of diffusion tensor imaging

- and $1 \mathrm{H}$ spectroscopy for outcome prediction in severe traumatic brain injury: preliminary results. Crit Care Med 2009; 37:1448-1455.

Important study on the use of structural MRI disentangling bad from good outcome after brain trauma and coma in adults, awaiting the results from ongoing larger multicentric studies.

37 Fernandez-Espejo D, Junque C, Cruse D, et al. Combination of diffusion tensor and functional magnetic resonance imaging during recovery from the vegetative state. BMC Neurol 2010; 10:77.

38 Machado C, Rodriguez R, Carballo M, et al. Brain anatomy, cerebral blood flow, and connectivity in the transition from PVS to MCS. Rev Neurosci 2009 20:177-180.

39 Aaen GS, Holshouser BA, Sheridan C, et al. Magnetic resonance spectro-

- $\quad$ scopy predicts outcomes for children with nonaccidental trauma. Pediatrics $2010 ; 125: 295-303$.

Important study on the use of structural MRI disentangling bad from good outcome after brain trauma and coma in children.

$40 \mathrm{Di} \mathrm{H}$, Boly M, Weng X, et al. Neuroimaging activation studies in the vegetative state: predictors of recovery? Clin Med 2008; 8:502-507.

41 Coleman MR, Davis MH, Rodd JM, et al. Towards the routine use of brain

- imaging to aid the clinical diagnosis of disorders of consciousness. Brain 2009; 132:2541-2552.

Milestone series on the use of activation fMRI studies in predicting outcome from vegetative state/UWS and minimally conscious state patients.

42 Bagnato S, Boccagni C, Prestandrea C, et al. Prognostic value of standard EEG in traumatic and nontraumatic disorders of consciousness following coma. Clin Neurophysiol 2010; 121:274-280.

43 Babiloni C, Sara M, Vecchio F, et al. Cortical sources of resting-state alpha rhythms are abnormal in persistent vegetative state patients. Clin Neurophysiol 2009; 120:719-729.

44 Cologan V, Schabus M, Ledoux D, et al. Sleep in disorders of consciousness. - $\quad$ Sleep Med Rev 2010; 14:97-105.

Exhaustive review on the understudied field of EEG assessment of sleep in coma and related disorders.

45 Cavinato M, Freo U, Ori C, et al. Postacute P300 predicts recovery of consciousness from traumatic vegetative state. Brain Inj 2009; 23:973980.

46 Bekinschtein TA, Shalom DE, Forcato $C$, et al. Classical conditioning in the

-. vegetative and minimally conscious state. Nat Neurosci 2009; 12:13431349.

Landmark neuroscience article showing associative learning (conditioning) in some cases of 'vegetative' state.

47 Laureys S. The neural correlate of (un)awareness: lessons from the vegetative state. Trends Cogn Sci 2005; 9:556-559.
48 Murphy M, Bruno MA, Riedner B, et al. Propofol anesthesia and sleep: a high- density EEG study. Sleep (in press)

Using high-density EEG and cortical source modeling, this article shows that pharmacological loss of consciousness (in propofol anesthesia) differs from the fading consciousness during sleep. Results from ongoing studies using this approach in pathological unconsciousness and coma are awaited.

49 Noirhomme $\mathrm{Q}$, Boly $\mathrm{M}$, Bonhomme V, et al. Bispectral index correlates with regional cerebral blood flow during sleep in distinct cortical and subcortical structures in humans. Arch Ital Biol 2009; 147:51-57.

50 Vanhaudenhuyse $\mathrm{A}$, Boly $\mathrm{M}$, Balteau $\mathrm{E}$, et al. Pain and nonpain processing during hypnosis: a thulium-YAG event-related fMRI study. Neuroimage 2009; 47:1047-1054.

51 Demertzi A, Soddu A, Faymonville M-E, et al. Hypnotic modulation of resting state fMRI default mode and extrinsic network connectivity. Prog Brain Res (in press).

52 Boveroux $\mathrm{P}$, Vanhaudenhuyse $\mathrm{A}$, Bruno MA, et al. Breakdown of within- and

- between-network resting state functional magnetic resonance imaging connectivity during propofol-induced loss of consciousness. Anesthesiology 2010; 113:1038-1053.

An anesthesia study demonstrating the critical role of thalamocortical connectivity in the genesis of conscious perception concluding that both within-network and between-network connectivity and communication between low-level sensory and higher-order frontoparietal cortices are necessary for perception of external sensory stimuli.

53 Lapitskaya N, Coleman MR, Nielsen JF, et al. Disorders of consciousness: further pathophysiological insights using motor cortex transcranial magnetic stimulation. Prog Brain Res 2009; 177:191-200.

54 Silva S, Alacoque X, Fourcade O, et al. Wakefulness and loss of awareness: brain and brainstem interaction in the vegetative state. Neurology 2010; $74: 313-320$

55 Lull N, Noe E, Lull JJ, et al. Voxel-based statistical analysis of thalamic glucose

- metabolism in traumatic brain injury: relationship with consciousness and cognition. Brain Inj 2010; 24:1098-1107.

Important PET study confirming the role of metabolic thalamic dysfunction in DOC.

56 Fernandez-Espejo D, Junque C, Bernabeu M, et al. Reductions of thalamic

- volume and regional shape changes in the vegetative and the minimally conscious states. J Neurotrauma 2010; 27:1187-1193.

Important MRI study highlighting the role of structural thalamic damage in DOC.

57 Demertzi A, Liew C, Ledoux D, et al. Dualism persists in the science of mind. Ann N Y Acad Sci 2009; 1157:1-9.

58 Coleman MR, Bekinschtein T, Monti MM, et al. A multimodal approach to the assessment of patients with disorders of consciousness. Prog Brain Res 2009; 177:231-248.

59 Schiff ND. Recovery of consciousness after brain injury: a mesocircuit hypothesis. Trends Neurosci 2010; 33:1-9.

60 Demertzi A, Vanhaudenhuyse A, Bruno MA, et al. Is there anybody in there? Detecting awareness in disorders of consciousness. Expert Rev Neurother 2008; 8:1719-1730.

61 Schnakers C, Hustinx R, Vandewalle G, et al. Measuring the effect of amantadine in chronic anoxic minimally conscious state. J Neurol Neurosurg Psychiatry 2008; 79:225-227.

62 Kim YW, Shin JC, An YS. Effects of methylphenidate on cerebral glucose metabolism in patients with impaired consciousness after acquired brain injury. Clin Neuropharmacol 2009; 32:335-339.

63 Fins JJ. Neuroethics and neuroimaging: moving toward transparency. Am J Bioeth 2008; 8:46-52.

64 Schiff ND, Giacino JT, Fins JJ. Deep brain stimulation, neuroethics, and the minimally conscious state: moving beyond proof of principle. Arch Neurol 2009; 66:697-702.

65 Fins JJ, Schiff ND. Conflicts of interest in deep brain stimulation research and the ethics of transparency. J Clin Ethics 2010; 21:125-132. 